University of Nebraska - Lincoln

DigitalCommons@University of Nebraska - Lincoln

John R. Hardy Papers

Research Papers in Physics and Astronomy

$1-15-1969$

\title{
Application of the Method of Lattice Statics to Vacancies in $\mathrm{Na}, \mathrm{K}$, $\mathrm{Rb}$, and $\mathrm{Cs}$
}

John W. Flocken

University of California, Livermore, California

John R. Hardy

University of Nebraska - Lincoln

Follow this and additional works at: https://digitalcommons.unl.edu/physicshardy

Part of the Physics Commons

Flocken, John W. and Hardy, John R., "Application of the Method of Lattice Statics to Vacancies in Na, K, Rb, and Cs" (1969). John R. Hardy Papers. 8.

https://digitalcommons.unl.edu/physicshardy/8

This Article is brought to you for free and open access by the Research Papers in Physics and Astronomy at DigitalCommons@University of Nebraska - Lincoln. It has been accepted for inclusion in John R. Hardy Papers by an authorized administrator of DigitalCommons@University of Nebraska - Lincoln. 


\title{
Application of the Method of Lattice Statics to Vacancies in $\mathrm{Na}, \mathrm{K}, \mathrm{Rb}$, and $\mathrm{Cs}^{*}$
}

\author{
J. W. Flocken† aNd J. R. HaRdy \\ Lawrence Radiation Laboratory, University of California, Livermore, California 94550
}

(Received 5 August 1968)

\begin{abstract}
We have calculated the lattice distortion produced by a single vacancy in $\mathrm{Na}, \mathrm{K}, \mathrm{Rb}$, and $\mathrm{Cs}$. The calculations have been carried out using the technique of lattice statics, which is based on the Fourier transformation of the direct-space equilibrium equations, making consistent use of discrete lattice theory. Three distinct types of potential have been used to describe the interactions between the host atoms. The first of these applies only to sodium, and contains an ion-electron-ion term derived from the measured phonon dispersion curves. The second applies only to potassium, and has been similarly obtained. The third is based on a model pseudopotential and applies to all four metals. Comparison has been made between our displacements due to a single vacancy in $\mathrm{Na}$, using the first of these potentials, and analogous results obtained by a semidiscrete method in which only the atoms in the first five shells are allowed to relax. The agreement is reasonable for atoms in the first two neighbor shells about the vacancy, but poor for atoms farther away. The calculated displacements have been used to calculate the dilatations and relaxation energies associated with single vacancies in alkali metals. There is a large discrepancy between the magnitudes of these quantities calculated using the first $\mathrm{Na}$ potential and those obtained using the second $\mathrm{Na}$ potential, and a similar discrepancy exists between the two sets of $\mathrm{K}$ results. We have also used the method of lattice statics to determine the strain-field interaction energies between several types of vacancy pairs in these metals. In every case we find the next-nearest-neighbor configuration to be the most stable, whereas in the nearest-neighbor configuration, the two vacancies repel one another. The magnitudes of these binding energies depend strongly on which model potential is used.
\end{abstract}

\section{INTRODUCTION}

$\mathrm{P}$ OINT defects can be introduced into a crystal lattice in two distinct ways; they can be generated by thermal activation or by fast particle irradiation. In order to understand theoretically the behavior of defects generated by either method, it is of basic importance to be able to calculate accurately the formation energies of the various species of defect produced and also to be able to calculate the binding energies of various defect pairs. In the case of simple crystals such as the alkali metals, which are our concern in the present paper, the simplest type of defect is the lattice vacancy.

The first stage in understanding the defect properties of these materials is understanding the properties of a single isolated vacancy in one of them. To do this, it is essential that we have some means of calculating precisely the lattice configuration about such a vacancy. This is important both in its own right insofar as it plays an important role in such properties as the electrical resistivity induced by the defect, and also because it is essential to know the macroscopic volume change associated with the formation since this plays a critical role in determining the actual formation energy of the defect.

To solve these problems one needs first a realistic interatomic potential for the atoms in the metal and second, given such a potential, a means of calculating rigorously the minimum energy configuration when a vacancy is introduced into the lattice.

\footnotetext{
* Work performed in part under the auspices of the U. S. Atomic Energy Commission.

$\dagger$ Permanent address: Behlen Laboratory of Physics, University of Nebraska, Lincoln, Neb.

† Summer visiting Professor of Physics. Permanent address: Behlen Laboratory of Physics, University of Nebraska, Lincoln, Neb.
}

A recent calculation of the distortion produced by a single vacancy in sodium has been performed by Shyu et al. ${ }^{1}$ using an interatomic potential due to Cochran. ${ }^{2}$ The method of computation used was typical of the class of techniques which might be referred to as "semidiscrete" methods. This classification we apply to any approach in which the ions in a predefined region near the defect, which we will call Region I, are allowed to relax to new equilibrium positions without further constraint. The remaining atoms in the lattice are then either held fixed or forced to relax to positions determined by continuum elasticity theory. If Region I can be made large enough, accurate displacements can be obtained for any host atom. However, the equations governing the displacements of the atoms of Region I increase rapidly in number and complexity as the volume of Region I increases; thus in practice, the size of this region is rather severely limited.

In a recent paper $^{3}$ we demonstrated the inadequacy of the "semidiscrete" methods which have been applied to the problem of the body-centered interstitial copper atom in a copper lattice, even though the forces exerted on the host atom by the defect extended only to nearest neighbors. In the case of the alkali metals such methods are likely to be even more inapplicable since in these metals the range of the interatomic potential is known from neutron scattering to extend at least to fifth neighbors. It is the purpose of the present work to determine the distortion produced by a single vacancy in the alkali metals $\mathrm{Na}, \mathrm{K}, \mathrm{Rb}$, and $\mathrm{Cs}$, by the consistent application of discrete lattice theory, without the neces-

\footnotetext{
${ }^{1}$ W. M. Shyu, D. Brust, and F. G. Fumi, J. Phys. Chem. Solids 28, 717 (1967).

${ }^{2}$ W. Cochran, Proc. Roy. Soc. (London) A276, 308 (1963).

${ }^{3} \mathrm{~J}$. W. Flocken and J. R. Hardy (to be published).
} 
sity at any stage of using continuum elasticity. This same technique of lattice statics also allows us to determine the strain-field interaction energy between pairs of vacancies.

This method was originally developed by $\mathrm{Kanzaki}^{4}$ and has been further applied and extended by Hardy. ${ }^{5}$ This approach is based on calculating the real space displacements of the various atoms in the imperfect lattice by Fourier transforming these displacements and first determining their Fourier components from the Fourier transformed equilibrium equations.

We determine the allowed wave vectors by applying periodic boundary conditions across a supercell consisting of $N$ primitive unit cells. This is equivalent to treating a superlattice of defects, one in each supercell. The Fourier transformation reduces the $3 N \times 3 N$ array of linear equations which determines the direct space displacements to a system of $N 3 \times 3$ equations which are explicitly soluble.

We will first apply the method of lattice statics to the problem of the single vacancy in $\mathrm{Na}$, using the force constants derived from the potential of Shyu et al., ${ }^{1}$ which will allow us to compare our final displacements with theirs. These calculations will be repeated for $\mathrm{Na}$ and extended to $\mathrm{K}, \mathrm{Rb}$, and $\mathrm{Cs}$ also, using the force constants calculated by Shyu and Gaspari ${ }^{6}$ derived from the results of Heine and co-workers ${ }^{7,8}$ on model pseudopotential form factors. In this work, a modified Hartree dielectric function is used to allow for the screening of the bare pseudopotential by the conduction electrons. Finally, we will repeat our calculations for $\mathrm{K}$ using force constants given by Cowley et $a l .{ }^{9}$ obtained by a least-squares fit to their experimental phonon dispersion curves.

In Sec. II we present the basic equations of lattice statics appropriate to the calculation of the displacement field due to a single defect, as well as the equations governing the interaction between pairs of defects. In Sec. III we present detailed calculations of the displacement field about a single vacancy in $\mathrm{Na}, \mathrm{K}, \mathrm{Rb}$, and $\mathrm{Cs}$ and the analogous calculations of the strain field interaction energies between pairs of vacancies. In Sec. IV we calculate the dilatations produced by single vacancies and their associated relaxation energies. Section $\mathrm{V}$ will be devoted to a comparison of our results with those of Shyu et al. ${ }^{1}$ and to a general discussion of all of our calculations.

\section{METHOD OF LATTICE STATICS}

The complete derivation of the equations necessary to the solution of a problem by the method of lattice

${ }^{4}$ H. Kanzaki, J. Phys. Chem. Solids 2, 24 (1957).

5 J. R. Hardy, J. Phys. Chem. Solids 15, 39 (1960).

${ }^{6}$ W. M. Shyu and G. D. Gaspari, Phys. Rev. 163, 667 (1967).

${ }^{7}$ V. Heine and I. Abarenkov, Phil. Mag. 9, 451 (1964).

8 A. O. E. Animalu and V. Heine, Phil. Mag. 12, 1249 (1965)

9 R. A. Cowley, A. D. B. Woods, and G. Dolling, Phys. Rev. 150, 487 (1966).
TABLE I. Force constant matrix forms for bcc metals.

\begin{tabular}{|c|c|}
\hline Neighbor & Force constant matrix \\
\hline 111 & $\left(\begin{array}{lll}\alpha_{1} & \beta_{1} & \beta_{1} \\
\beta_{1} & \alpha_{1} & \beta_{1} \\
\beta_{1} & \beta_{1} & \alpha_{1}\end{array}\right)$ \\
\hline 200 & $\left(\begin{array}{lll}\alpha_{2} & 0 & 0 \\
0 & \beta_{2} & 0 \\
0 & 0 & \beta_{2}\end{array}\right)$ \\
\hline 220 & $\left(\begin{array}{ccc}\alpha_{3} & \gamma_{3} & 0 \\
\gamma_{3} & \alpha_{3} & 0 \\
0 & 0 & \beta_{3}\end{array}\right)$ \\
\hline 311 & $\left(\begin{array}{ccc}\alpha_{4} & \gamma_{4} & \gamma_{4} \\
\gamma_{4} & \beta_{4} & \delta_{4} \\
\gamma_{4} & \delta_{4} & \beta_{4}\end{array}\right)$ \\
\hline 222 & $\left(\begin{array}{lll}\alpha_{5} & \beta_{5} & \beta_{5} \\
\beta_{5} & \alpha_{5} & \beta_{5} \\
\beta_{5} & \beta_{5} & \alpha_{5}\end{array}\right)$ \\
\hline
\end{tabular}

statics has been given. ${ }^{3}$ We will not repeat this discussion in detail, but will simply present a brief outline of the principles upon which the method is based, along with the equations necessary to perform the present calculations.

Let $\mathbf{a}_{1}, \mathbf{a}_{2}$, and $\mathbf{a}_{3}$ be the basis vectors of an infinite lattice which we suppose to be built up from equivalent volumes containing $N$ unit cells. The boundaries of these volumes, called supercells, may be taken as being $L$ times the basic vectors where $L^{3}=N$.

At the center of each supercell we imagine a defect to be introduced. Symmetry will then require that the displacements of the atoms around any one defect will be identical to those of the corresponding atoms about any other defect. Hence we need only treat the atoms of a single supercell in order to find the displacement of any other atom in the crystal.

The solution of the displacement problem is obtained by expressing the displacement of the $l$ th atom from the origin in terms of normal coordinates $\mathbf{Q}^{\mathrm{q}}$ using the Fourier series

$$
\zeta_{\alpha}^{l}=\frac{1}{N} \sum_{\mathbf{q}} \mathbf{q}_{\alpha^{\mathrm{q}}} e^{i \mathrm{q} \cdot \mathbf{r}^{(l)}},
$$

where $\mathbf{q}$ is an allowed wave vector in the first Brillouin zone and $\boldsymbol{r}^{(l)}$ is the position vector of the $l$ th atom from the origin in direct space in the undistorted lattice. The parameter $\alpha$ denotes the Cartesian component of $\zeta^{l}$ or $\mathbf{Q}^{\mathrm{q}}$ along one of the three orthogonal $\langle 100\rangle$ directions in the cubic crystal and may therefore take on values 1,2 , 3. The value of $\mathbf{Q}^{\mathrm{q}}$ is found from the equation

$$
\mathbf{Q}^{\mathrm{q}}=\left(\mathbf{V}^{-\mathrm{q}}\right)^{-1} \mathbf{F} \mathrm{q},
$$

where $\mathbf{V}^{-\mathbf{q}}$ is the Fourier-transformed dynamical matrix and $\mathbf{F}^{\mathrm{q}}$ is the generalized force array associated with the defect. In terms of direct space quantities, these are 
TABLE II. Force constants for $\mathrm{Na}, \mathrm{K}, \mathrm{Rb}, \mathrm{Cs}$, used for displacement and interaction energy calculations.

\begin{tabular}{|c|c|c|c|c|c|c|}
\hline \multirow{2}{*}{$\begin{array}{c}\text { Force } \\
\text { constant } \\
\text { (dyn } / \mathrm{cm})\end{array}$} & \multicolumn{5}{|c|}{ Metal } & \multirow[b]{2}{*}{$\begin{array}{c}\mathrm{Cs}^{\mathrm{b}} \\
a=3.025 \AA\end{array}$} \\
\hline & $\begin{array}{c}\mathrm{Na}(1) \mathrm{s} \\
a=2.14 \AA\end{array}$ & $\begin{array}{c}\mathrm{Na}(2)^{\mathrm{b}} \\
a=2.14 \AA\end{array}$ & $\begin{array}{c}\mathrm{K}(1)^{\mathrm{c}} \\
a=2.665 \AA\end{array}$ & $\begin{array}{c}\mathrm{K}(2)^{\mathrm{b}} \\
a=2.665 \AA\end{array}$ & $\begin{array}{c}\mathrm{Rb}^{\mathrm{b}} \\
a=2.81 \AA\end{array}$ & \\
\hline$\alpha_{1}$ & 1123.30 & 1656.08 & 786 & 903.67 & 692.78 & 563.41 \\
\hline$\beta_{1}$ & 1290.90 & 2099.44 & 895 & 1280.09 & 1039.38 & 862.39 \\
\hline$\alpha_{2}$ & 445.50 & 1431.53 & 432 & 1181.10 & 1072.97 & 933.14 \\
\hline$\beta_{2}$ & 94.60 & 64.48 & 29 & -29.48 & -50.71 & -48.14 \\
\hline$\alpha_{3}$ & -45.40 & -128.25 & -41 & -70.70 & -52.61 & -44.365 \\
\hline$\beta_{3}$ & -9.30 & 15.74 & 12 & 14.30 & 12.85 & 11.33 \\
\hline$\gamma_{3}$ & -36.10 & -143.99 & -54 & -85.00 & -65.46 & -55.695 \\
\hline$\alpha_{4}$ & 55.50 & $-\quad 3.12$ & 2 & -26.74 & -28.41 & -25.93 \\
\hline$\beta_{4}$ & 3.50 & -2.64 & -4 & -3.58 & -3.068 & -2.72 \\
\hline$\gamma_{4}$ & 19.5 & $-\quad 0.18$ & 1.8 & -8.70 & -9.51 & -8.70 \\
\hline$\delta_{4}$ & 6.5 & $-\quad 0.06$ & 0.6 & -2.90 & -3.17 & -2.901 \\
\hline$\alpha_{5}$ & 14.50 & 8.75 & 6 & -3.15 & -4.87 & -5.047 \\
\hline$\beta_{5}$ & 14.90 & 10.57 & 4 & -1.68 & -3.95 & -4.283 \\
\hline
\end{tabular}

a Reference 1.

defined by

$$
F_{\alpha}^{\mathrm{q}} \equiv \sum_{l} F_{\alpha}^{l} e^{-i \mathrm{q} \cdot \mathrm{r}^{(l)}}
$$

and

$$
V_{\alpha \beta}{ }^{-\mathrm{q}} \equiv \sum_{l^{\prime}} V_{\alpha \beta} 0 l^{\prime} e^{i \mathrm{q} \cdot \mathbf{r}^{\left(l^{\prime \prime}\right)}} .
$$

Once $\mathbf{F q}^{\mathrm{q}}$ and $\mathbf{V}^{-\mathbf{q}}$ have been calculated from Eqs. (3) and (4) we can compute the Fourier amplitudes $\mathbf{Q}^{\mathrm{q}}$ from Eq. (2) and perform the summation in Eq. (1) to obtain the direct space displacements. Similarly, it has been shown ${ }^{10}$ that the strain-field interaction energy between a pair of defects is given by

$$
E=-\frac{1}{N} \sum_{\mathbf{q}} \mathbf{F}^{-\mathbf{q}}\left(\mathbf{V}^{-\mathbf{q}}\right)^{-1} \mathbf{F q} \cos (\mathbf{q} \cdot \mathbf{R}),
$$

in which $\mathbf{R}$ is the distance between defect sites in the perfect lattice.

Since we are considering only vacancies in the present work, we may construct the dynamical matrix $\mathbf{V}^{-\mathbf{q}}$ and the force array $\mathbf{F}$ without using an explicit expression for the interatomic potential. As we will show shortly, it will be sufficient to know the force constants for the perfect lattice subject to the assumption that these are derivable from some central pairwise potential, as is the case for each of the potentials used in the numerical calculations. We shall express the elements of the matrix $\mathbf{V}^{-\mathbf{q}}$ in terms of the elements of the direct space interatomic force constant matrices which are presented in Table I. We assume in all cases that the atoms interact with each other as far as the fifth-neighbor positions. It has been shown ${ }^{6}$ that this assumption is adequate for $\mathrm{Na}, \mathrm{K}, \mathrm{Rb}$, and $\mathrm{Cs}$. For $\mathrm{Li}$, however, a much longer range potential is needed, extending as far as the tenthneighbor shell. Because of the additional computational difficulties which this long-range potential involves, we will not include $\mathrm{Li}$ in the present calculations.

\footnotetext{
${ }^{10}$ J. R. Hardy and R. Bullough, Phil. Mag. 15, 237 (1967).
}

We shall denote the magnitude of the force exerted by a single vacancy on the members of its $n$ th-neighbor shell as $F(n)$ where, as we have indicated, $n$ may range from 1 through 5.

With the notation we have outlined above, the components of the $\mathbf{V}^{-\mathbf{q}}$ matrix may be expressed as

$$
\begin{gathered}
V_{11^{-q}}=4 \beta_{2}+2 \alpha_{2}-2 \beta_{2}\left\{\cos 2 a q_{2}+\cos 2 a q_{3}\right\}-2 \alpha_{2} \cos 2 a q_{1} \\
-8 \alpha_{1}\left\{\cos q_{1} a \cos q_{2} a \cos q_{3} a-1\right\}+8 \alpha_{3}+4 \beta_{3} \\
-4 \alpha_{3} \cos 2 q_{1} a\left(\cos 2 q_{2} a+\cos 2 q_{3} a\right)-4 \beta_{3} \cos 2 q_{2} a \cos 2 q_{2} a \\
+8 \alpha_{4}-8 \alpha_{4} \cos 3 q_{1} a \cos q_{2} a \cos q_{3} a+16 \beta_{4} \\
-8 \beta_{4} \cos q_{1} a\left(\cos q_{3} a \cos 3 q_{2} a+\cos q_{2} a \cos 3 q_{3} a\right) \\
-8 \alpha_{5}\left(\cos 2 q_{1} a \cos 2 q_{2} a \cos 2 q_{3} a-1\right)
\end{gathered}
$$

and

$V_{12}-\mathrm{q}=8 \beta_{1} \sin q_{1} a \sin q_{2} a \cos q_{3} a+4 \gamma_{3} \sin 2 q_{1} a \sin 2 q_{2} a$ $+8 \gamma_{4}\left(\sin 3 q_{1} a \cos q_{3} a \sin q_{2} a+\sin 3 q_{2} a \cos q_{3} a \sin q_{1} a\right)$

$-8 \delta_{4}\left(\cos 3 q_{3} a \sin q_{1} a \sin q_{2} a\right)$ $+8 \beta_{5} \sin 2 q_{1} a \sin 2 q_{2} a \cos 2 q_{3} a$.

The remaining elements of $V^{q}$ can be obtained by cyclic permutation of the $\mathbf{q}$ vector components. The $\mathrm{F}^{\mathrm{q}}$ array can be shown to be given by

$$
\begin{aligned}
& F_{1}{ }^{q}=-[8 i F(1) / \sqrt{3}] \sin q_{1} a\left\{\cos q_{2} a \cos q_{3} a\right\} \\
& -4 i F(2) \sin q_{1} a \cos q_{1} a \\
& -2 \sqrt{2} i F(3) \sin 2 q_{1} a\left(\cos 2 q_{2} a+\cos 2 q_{3} a\right) \\
& -[24 i F(4) / \sqrt{ } 11] \sin 3 q_{1} a \cos q_{2} a \cos q_{3} a \\
& -[8 i F(4) / \sqrt{ } 11] \sin q_{1} a \\
& \quad \times\left\{\cos q_{2} a \cos 3 a q_{3}+\cos q_{3} a \cos 3 q_{2} a\right\} \\
& \quad-[8 i F(5) / \sqrt{3}] \sin 2 q_{1} a\left\{\cos 2 q_{2} a \cos 2 q_{3} a\right\},
\end{aligned}
$$

and two similar equations for $F_{2} \mathrm{q}$ and $F_{3} \mathrm{q}$ can be obtained by permuting the components of $\mathbf{q}$.

All that remains to be done is to find appropriate expressions for the forces $F(n)$ in terms of the direct space-force constants. In practice it is easiest to work 
TABLE III. Displacement and interaction energy results for $\mathrm{Na}(1) .^{\mathrm{a}}$

\begin{tabular}{|c|c|c|c|c|c|}
\hline \multirow{2}{*}{$\begin{array}{l}\text { Neighbor } \\
\left(L_{1}, L_{2}, L_{3}\right)\end{array}$} & \multirow[t]{2}{*}{$\begin{array}{c}\text { Interaction } \\
\text { energy in } \mathrm{eV} \\
\text { between }(0,0,0) \\
\text { and }\left(L_{1}, L_{2}, L_{3}\right) \\
\text { vacancies }\end{array}$} & \multicolumn{3}{|c|}{$\begin{array}{l}\text { Displacement components of neighbors } \\
\text { around a vacancy (units of } 2 a \text { ) }\end{array}$} & \multirow[t]{2}{*}{$\begin{array}{c}|\zeta|\left\{L_{1}{ }^{2}+L_{2}{ }^{2}+L_{3}{ }^{2}\right\} \\
\text { + indicates } \\
\text { outward } \\
\text { relaxation } \\
\text { - indicates } \\
\text { inward } \\
\text { relaxation }\end{array}$} \\
\hline & & $\zeta_{1}$ & $\zeta_{2}$ & $\zeta_{3}$ & \\
\hline 111 & 0.006282 & -0.02935 & -0.02935 & -0.02935 & -0.1525 \\
\hline 200 & -0.01502 & 0.03180 & 0.0 & 0.0 & +0.1272 \\
\hline 220 & 0.01471 & -0.009550 & -0.009550 & 0.0 & -0.1080 \\
\hline 222 & 0.007986 & -0.01434 & -0.01434 & -0.01434 & -0.2980 \\
\hline 311 & -0.01262 & 0.004580 & 0.001854 & 0.001854 & +0.05805 \\
\hline 331 & 0.007007 & -0.004977 & -0.004977 & -0.000528 & -0.1341 \\
\hline 333 & 0.004059 & -0.007660 & -0.007660 & -0.007660 & -0.3582 \\
\hline 400 & 0.003882 & 0.001954 & 0.0 & 0.0 & +0.03127 \\
\hline 420 & -0.003006 & 0.0005935 & 0.0002243 & 0.0 & +0.01269 \\
\hline 422 & -0.004393 & 0.001156 & -0.0003015 & -0.0003015 & +0.02957 \\
\hline 440 & 0.002631 & -0.003064 & -0.003064 & 0.0 & -0.1387 \\
\hline 442 & 0.002959 & -0.002949 & -0.002949 & -0.0006096 & -0.1517 \\
\hline 444 & 0.002311 & -0.004261 & -0.004261 & -0.004261 & -0.3542 \\
\hline 511 & -0.0001439 & 0.001027 & -0.00001615 & -0.00001615 & +0.02773 \\
\hline 531 & -0.001022 & -0.00006627 & -0.0002428 & -0.0001838 & -0.01091 \\
\hline 533 & -0.001809 & -0.0001472 & -0.0008395 & -0.0008395 & -0.05144 \\
\hline 551 & 0.001442 & -0.001968 & -0.001968 & -0.0002138 & -0.1424 \\
\hline 555 & 0.001288 & -0.002492 & -0.002492 & -0.002492 & +0.3237 \\
\hline 600 & -0.0005302 & 0.0008190 & 0.0 & 0.0 & +0.02949 \\
\hline 620 & -0.0002979 & 0.0005104 & 0.00009950 & 0.0 & +0.02080 \\
\hline 622 & -0.0002615 & 0.0004182 & -0.0001857 & -0.0001857 & +0.02173 \\
\hline 640 & -0.0004292 & -0.0002477 & -0.0003623 & 0.0 & -0.02282 \\
\hline
\end{tabular}

a Using Ref. 1 force constants.

with radial and tangential force constants $A(n)$ and $B(n)$ defined by

$$
\begin{aligned}
& A(n)=2\left(\partial^{2} \psi / \partial r^{2}\right)_{r(n)}=2 \psi^{\prime \prime}(n), \\
& B(n)=2((1 / r)(\partial \psi / \partial r))_{r(n)}=[2 / r(n)] \psi^{\prime}(n),
\end{aligned}
$$

where $\psi(r)$ is the interatomic potential acting between host atoms, so that $-\psi(r)$ is the potential acting between a vacancy and the host atoms. The term $r(n)$ is the radius of the $n$ th-neighbor shell in direct space, in the perfect lattice.

Our method of determining the forces $F(n)$ makes it unnecessary to know $\psi(r)$ explicitly, but only requires a knowledge of the direct-space force constants shown in Table I. Then $A(n)$ and $B(n)$ are related to these constants by the following equations:

$$
\begin{array}{ll}
A(1)=2\left(\alpha_{1}+2 \beta_{1}\right) & B(1)=-2\left(\beta_{1}-\alpha_{1}\right) \\
A(2)=2\left(\alpha_{2}\right) & B(2)=2 \beta_{2} \\
A(3)=2\left(\alpha_{3}+\gamma_{3}\right) & B(3)=2 \beta_{3} \\
A(4)=\left(6 \alpha_{4}+4 \gamma_{4}\right) / 3 & B(4)=2\left(\alpha_{4}-3 \gamma_{4}\right) \\
A(5)=2\left(\alpha_{5}+2 \beta_{5}\right) & B(5)=-2\left(\beta_{5}-\alpha_{5}\right) .
\end{array}
$$

In terms of the notation just developed

$$
F(n)=\psi^{\prime}(|\mathbf{r}(n)+\zeta(n)|),
$$

where the derivative $\psi^{\prime}$ is evaluated at the relaxed posi- tion of the $n$ th-neighbor shell. We can avoid any need to know $\psi(r)$ explicitly by linearizing the $F(n)$ in the lattice displacements. Thus

$F(n)=\psi^{\prime}(n)+\zeta(n) \psi^{\prime \prime}(n)=\frac{1}{2} r(n) B(n)+\zeta(n) \frac{1}{2} A(n)$,

in which $\zeta(n)$ is the magnitude of the displacements of the atoms in the $n$th shell. These may be expressed as follows:

$$
\zeta(n)=\sum_{m} \rho(n, m) F(m)
$$

The coefficients $\rho(n, m)$ are the elements of a response matrix obtained by the inversion of the Fourier series (1), i.e., $\rho(n, m)$ is the magnitude of the displacement of an atom in the $n$th shell when the $m$ th force is unity and all other forces are zero. Since we are allowing for up to fifth-neighbor interactions, $m$ and $n$ range from 1 through 5 . Once the $\rho(n, m)$ are known, one may solve Eqs. (12) and (13) for the displacements $\zeta(n)$ which may then be used in (12) to find the forces $F(n)$. Using these values for the forces $F(n)$, one can compute the corresponding generalized forces $\mathrm{Fa}^{\mathrm{q}}$ from $\mathrm{Eq}$. (8).

Since the displacements of the fourth- and fifthneighbor shells are not expected to be large, we will approximate the forces $F(4)$ and $F(5)$ by their values at the undisplaced positions. 
TABLE IV. Displacement and interaction energy results for $\mathrm{Na}(2) .^{\mathrm{a}}$

\begin{tabular}{|c|c|c|c|c|c|}
\hline $\begin{array}{l}\text { Neighbor } \\
\left(L_{1}, L_{2}, L_{3}\right)\end{array}$ & $\begin{array}{c}\text { Interaction } \\
\text { energy in } \mathrm{eV} \\
\text { between }(0,0,0) \\
\text { and }\left(L_{1}, L_{2}, L_{3}\right) \\
\text { vacancies }\end{array}$ & \multicolumn{3}{|c|}{$\begin{array}{l}\text { Displacement components of neighbors } \\
\text { around a vacancy (units of } 2 a \text { ) }\end{array}$} & $\begin{array}{c}|\zeta|\left\{L_{1}^{2}+L_{2}^{2}+L_{3}^{2}\right\} \\
+ \text { indicates } \\
\text { outward } \\
\text { relaxation } \\
\text { - indicates } \\
\text { inward } \\
\text { relaxation }\end{array}$ \\
\hline 111 & 0.01535 & -0.05536 & -0.05536 & -0.05536 & -0.2877 \\
\hline 200 & -0.09935 & 0.05402 & 0.0 & 0.0 & +0.2161 \\
\hline 220 & 0.07420 & -0.01596 & -0.01596 & 0.0 & -0.1806 \\
\hline 222 & 0.04176 & -0.02992 & -0.02992 & -0.02992 & -0.6219 \\
\hline 311 & -0.05420 & 0.007334 & 0.002605 & 0.002605 & +0.09029 \\
\hline 331 & 0.02778 & -0.009074 & -0.009074 & -0.0003475 & -0.2440 \\
\hline 333 & 0.02614 & -0.01711 & -0.01711 & -0.01711 & -0.8002 \\
\hline 400 & 0.01005 & 0.006850 & 0.0 & 0.0 & +0.1096 \\
\hline 420 & -0.007106 & 0.0006615 & 0.0002765 & 0.0 & +0.01435 \\
\hline 422 & -0.02306 & 0.001964 & -0.001135 & -0.001135 & +0.06088 \\
\hline 440 & 0.01110 & -0.006183 & -0.006183 & 0.0 & -0.2798 \\
\hline 442 & 0.01231 & -0.005769 & -0.005769 & -0.0007287 & -0.2949 \\
\hline 444 & -0.01544 & -0.01007 & -0.01007 & -0.01007 & -0.8369 \\
\hline 511 & -0.002690 & 0.002659 & 0.0001093 & 0.0001093 & +0.07191 \\
\hline 531 & -0.003057 & -0.0002236 & -0.0004190 & -0.0002624 & -0.01899 \\
\hline 533 & -0.01025 & -0.0003968 & -0.002076 & -0.002076 & -0.1274 \\
\hline 551 & 0.006827 & -0.004180 & -0.004180 & -0.0003472 & -0.3020 \\
\hline 555 & 0.008915 & -0.006149 & -0.006149 & -0.006149 & -0.7988 \\
\hline 600 & -0.002725 & 0.002216 & 0.0 & 0.0 & +0.07979 \\
\hline 620 & -0.001129 & 0.001133 & 0.0003535 & 0.0 & +0.04746 \\
\hline 622 & -0.002177 & 0.001287 & -0.0003556 & -0.0003556 & +0.06079 \\
\hline 640 & -0.001862 & -0.0005497 & -0.0006650 & 0.0 & -0.04487 \\
\hline
\end{tabular}

a Using Ref. 6 force constants.

Thus,

while

$$
\begin{aligned}
& F(4)=(\sqrt{ } 11) a \frac{1}{2} B(4), \\
& F(5)=(\sqrt{ } 12) a \frac{1}{2} B(5),
\end{aligned}
$$

$$
\begin{aligned}
& F(1)=\sqrt{3} \frac{1}{2} a B(1)+\zeta(1) \frac{1}{2} A(1), \\
& F(2)=a B(2)+\zeta(2) \frac{1}{2} A(2), \\
& F(3)=\sqrt{2} a B(3)+\zeta(3) \frac{1}{2} A(3),
\end{aligned}
$$

where $a$ is half the cubic unit cell side.

Therefore, substituting from Eq. (14) into Eq. (12), we obtain

$$
\begin{aligned}
\zeta(1)= & \rho(1,1) \zeta(1) \frac{1}{2} A(1) \\
& +\rho(1,2) \zeta(2) \frac{1}{2} A(2)+\rho(1,3) \zeta(3) \frac{1}{2} A(3) \\
& +\rho(1,1) \sqrt{3} \frac{1}{2} a B(1)+\rho(1,2) a B(2)+\rho(1,3) \sqrt{2} a B(3) \\
& +\rho(1,4)(\sqrt{ } 11) \frac{1}{2} a B(4)+\rho(1,5)(\sqrt{ } 12) \frac{1}{2} a B(5), \\
\zeta(2)= & \rho(2,1) \zeta(1) \frac{1}{2} A(1)+\rho(2,2) \zeta(2) \frac{1}{2} A(2) \\
& +\rho(2,3) \zeta(3) \frac{1}{2} A(3)+\rho(2,1) \sqrt{3} \frac{1}{2} a B(1) \\
& +\rho(2,2) a B(2)+\rho(2,3) \sqrt{2} a B(3) \\
& +\rho(2,4)(\sqrt{ } 11) \frac{1}{2} a B(4)+\rho(2,5)(\sqrt{ } 12) \frac{1}{2} a B(5), \\
\zeta(3)= & \rho(3,1) \frac{1}{2} \zeta(1) A(1)+\rho(3,2) \frac{1}{2} \zeta(2) A(2) \\
& +\rho(3,3) \frac{1}{2} \zeta(3) A(3)+\rho(3,1) \sqrt{3} \frac{1}{2} a B(1)+\rho(3,2) a B(2) \\
& +\rho(3,3) \sqrt{2} a B(3)+\rho(3,4)(\sqrt{ } 11) \frac{1}{2} a B(4) \\
& \quad+\rho(3,5)(\sqrt{ } 12) \frac{1}{2} a B(5) .
\end{aligned}
$$

Once the displacements $\zeta(n)$ and the forces $F(n)$ are known, the Fourier amplitudes of the displacements can be calculated from Eq. (2). Then the direct-space com- ponents of displacement can be calculated from Eq. (1). Finally, the strain-field interaction energy can be computed using (5) with the appropriate values of the generalized force array $\mathbf{F}^{\mathrm{q}}$ calculated from Eq. (3).

\section{NUMERICAL CALCULATIONS AND RESULTS}

The present work is based upon interatomic potentials from three sources. The first potential used was that for $\mathrm{Na}$ metal given by Shyu $\mathrm{et} a \mathrm{al}{ }^{1}$ and derived from the work of Cochran. ${ }^{2}$ It consists of an overlap-repulsive term, a Coulombic ion-ion interaction, and an ionelectron-ion interaction which has been obtained from phonon dispersion curves. The second potential which we have used in our work is presented by Cowley et al. ${ }^{9}$ and is obtained by a least-squares fitting to the phonon dispersion curves measured at $9^{\circ} \mathrm{K}$. The third set of potentials is the result of theoretical calculations by Shyu and Gaspari carried out for $\mathrm{Li}, \mathrm{Na}, \mathrm{K}, \mathrm{Rb}$, and Cs. As stated earlier, we will not make use of their $\mathrm{Li}$ potential because its greater range increases the complexity of the problem beyond the scope of our present calculations.

These authors derived their potentials from pseudopotential form factors ${ }^{7,8}$ and a modified Hartree dielectric function. Since we will be treating $\mathrm{Na}$ and $\mathrm{K}$ using two distinct potentials for each metal, we will, for the sake of brevity, refer to the work using the potential of Ref. 1 as $\mathrm{Na}(1)$ and that using the potential of Ref. 6 as $\mathrm{Na}(2)$. Similarly, the $\mathrm{K}$ results obtained using the po- 
TABLE V. Displacement and interaction energy results for $K(1) .^{\mathbf{a}}$

\begin{tabular}{|c|c|c|c|c|c|}
\hline $\begin{array}{l}\text { Neighbor } \\
\left(L_{1}, L_{2}, L_{3}\right)\end{array}$ & $\begin{array}{c}\text { Interaction } \\
\text { energy in } \mathrm{eV} \\
\text { between }(0,0,0) \\
\text { and }\left(L_{1}, L_{2}, L_{3}\right) \\
\text { vacancies }\end{array}$ & \multicolumn{3}{|c|}{$\begin{array}{l}\text { Displacement components of neighbors } \\
\text { around a vacancy (units of } 2 a \text { ) }\end{array}$} & $\begin{array}{c}|\zeta|\left\{L_{1}^{2}+L_{2}^{2}+L_{3}{ }^{2}\right\} \\
\text { + indicates } \\
\text { outward } \\
\text { relaxation } \\
\text { - indicates } \\
\text { inward } \\
\text { relaxation }\end{array}$ \\
\hline & & -002551 & -002551 & -002551 & \\
\hline 111 & 0.007347 & $\begin{array}{r}-0.02551 \\
0.02281\end{array}$ & $\begin{array}{l}-0.02551 \\
0.0\end{array}$ & $\begin{array}{l}-0.02501 \\
0.0\end{array}$ & $\begin{array}{l}-0.1326 \\
+0.09122\end{array}$ \\
\hline $\begin{array}{l}200 \\
220\end{array}$ & $\begin{array}{r}-0.01833 \\
0.01068\end{array}$ & -0.005896 & -0.005896 & 0.0 & -0.06671 \\
\hline 222 & 0.007609 & -0.01260 & -0.01260 & -0.01260 & -0.2619 \\
\hline 311 & -0.008440 & 0.002400 & 0.0007299 & 0.0007299 & +0.02874 \\
\hline 331 & 0.002673 & -0.003783 & -0.003783 & 0.00004181 & -0.1017 \\
\hline 333 & 0.004180 & -0.006968 & -0.006968 & -0.006968 & -0.3259 \\
\hline 400 & 0.001627 & 0.002072 & 0.0 & 0.0 & +0.03316 \\
\hline 420 & -0.0001429 & 0.0001858 & 0.00001645 & 0.0 & +0.003730 \\
\hline 422 & -0.002843 & 0.0007030 & -0.0007538 & -0.0007538 & -0.03065 \\
\hline 440 & 0.001324 & -0.002671 & -0.002671 & 0.0 & -0.1209 \\
\hline 442 & 0.001286 & -0.002461 & -0.002461 & -0.0003672 & -0.1260 \\
\hline 444 & 0.002193 & -0.004010 & -0.004010 & -0.004010 & -0.3334 \\
\hline 511 & -0.0005115 & 0.0008795 & -0.0001315 & -0.0001315 & +0.02427 \\
\hline 531 & -0.0003331 & -0.0001071 & -0.0002534 & -0.0001537 & -0.01103 \\
\hline 533 & -0.001164 & -0.0002461 & -0.0009817 & -0.0009817 & -0.06063 \\
\hline 551 & 0.0008796 & -0.001787 & -0.001787 & -0.0001904 & -0.1293 \\
\hline 555 & 0.001174 & -0.002423 & -0.002423 & -0.002423 & -0.3147 \\
\hline 600 & -0.0005711 & 0.0008520 & 0.0 & 0.0 & +0.03067 \\
\hline 620 & -0.0001328 & 0.0004091 & 0.00003843 & 0.0 & +0.01643 \\
\hline 622 & -0.0002636 & 0.0003890 & -0.0002758 & -0.0002758 & +0.02424 \\
\hline 640 & -0.0002645 & -0.0002238 & -0.0003387 & 0.0 & -0.02111 \\
\hline
\end{tabular}

a Using Ref. 7 force constants.

tential of Ref. 9 will be called $\mathrm{K}(1)$ and the results obtained using the potential of Ref. 6 as $\mathrm{K}(2)$. The force constants derived from the various potentials for all of the cases we shall consider are shown in Table II.

In all of our calculations, we have used a sample of 64000 evenly spaced wave vectors in the first Brillouin zone. Our previous work on interstitial atoms in $\mathrm{Cu}$ leads us to believe this sample density is adequate to provide reliable displacement values out as far as $(5,4,1)$ and associated values of the strain-field interaction energy out at least as far as $(4,4,2)$.

The computations were all carried out on a CDC 3600 computer, and the resultant sets of displacements and defect interaction energies are presented in Tables III-VIII.

\section{CALCULATION OF DILATATIONS AND RELAXATION ENERGIES}

We calculate the dilatation according to the expression originally due to Eshelby, ${ }^{11}$

$$
\Delta V=G / K,
$$

in which $K$ is the bulk modulus and $G$ is the strength parameter which has been shown ${ }^{12}$ to be given by

$$
G=+\sum_{l} r_{\alpha} \frac{\partial \psi}{\partial \zeta_{\alpha}{ }^{l}}(|\mathbf{r}(l)+\zeta(l)|)=\sum_{l} r_{\alpha}^{l} F_{\alpha}^{l}
$$

${ }^{11}$ J. D. Eshelby, Solid State Phys. 3,79 (1956).

12 J. R. Hardy, J. Phys. Chem. Solids (to be published).
In this expression, $r_{\alpha}{ }^{l}$ is the $\alpha$ component of the direct space inter-defect spacing vector.

It is also of interest to determine the relaxation energy defined as the difference between the energy of the imperfect crystal before and after the atoms are allowed to relax to their equilibrium positions. This can be expressed as

$$
E_{R}=\psi(|\mathbf{r}(l)+\zeta(l)|)+\frac{1}{2} \tilde{\zeta} V \zeta-\psi(|\mathbf{r}(l)|),
$$

where $V$ is the force constant matrix for the metal in direct space. It can be shown that Eq. (18) reduces to

$$
E_{R}=\left.\frac{1}{2} \sum_{\alpha, l} \zeta_{\alpha}^{l} F_{\alpha}^{l}\right|_{0}
$$

provided one retains terms only up to second order in $\zeta$. The subscript 0 indicates that the forces are to be evaluated at the unrelaxed ion positions. The results of the calculations using Eqs. (16), (17), and (19) are shown in Table IX, where $\Delta V$ is the volume change due to taking one atom out of the crystal. Formation volumes are those shown in Table IX plus one atomic volume.

\section{DISCUSSION}

One of the important aspects of our calculations is that we may compare our results directly with those of Shyu et al..$^{1}$ These authors allowed the atoms in the first five shells around the vacancy to relax to new equilibrium positions under the influence of the forces arising from a single vacancy. The forces contributed from atoms in shells six through 18, i.e., those atoms 
TABLE VI. Displacement and interaction energy results for $\mathrm{K}(2)$.

\begin{tabular}{|c|c|c|c|c|c|}
\hline \multirow{2}{*}{$\begin{array}{l}\text { Neighbor } \\
\left(L_{1}, L_{2}, L_{3}\right)\end{array}$} & \multirow{2}{*}{$\begin{array}{c}\text { Interaction } \\
\text { energy in eV } \\
\text { between }(0,0,0) \\
\text { and }\left(L_{1}, L_{2}, L_{3}\right) \\
\text { vacancies }\end{array}$} & \multicolumn{3}{|c|}{$\begin{array}{l}\text { Displacement components of neighbors } \\
\text { around a vacancy (units of } 2 a \text { ) }\end{array}$} & \multirow[t]{2}{*}{$\begin{array}{c}|\zeta|\left\{L_{1}{ }^{2}+L_{2}{ }^{2}+L_{3}{ }^{2}\right\} \\
\text { + indicates } \\
\text { outward } \\
\text { relaxation } \\
\text { - indicates } \\
\text { inward } \\
\text { relaxation } \\
\end{array}$} \\
\hline & & $\zeta_{1}$ & $\zeta_{2}$ & $\zeta_{3}$ & \\
\hline 111 & 0.01820 & -0.09013 & -0.09013 & -0.09013 & -0.4684 \\
\hline 200 & -0.2271 & 0.09355 & 0.0 & 0.0 & +0.3742 \\
\hline 220 & 0.1642 & -0.02678 & -0.02678 & 0.0 & -0.3030 \\
\hline 222 & 0.08182 & -0.05186 & -0.05186 & -0.05186 & -1.078 \\
\hline 311 & -0.1220 & 0.01200 & 0.005335 & 0.005335 & +0.1559 \\
\hline 331 & 0.06419 & -0.01532 & -0.01532 & -0.0007852 & -0.4118 \\
\hline 333 & 0.05641 & -0.03094 & -0.03094 & -0.03094 & -1.447 \\
\hline 400 & 0.03249 & 0.01531 & 0.0 & 0.0 & +0.2450 \\
\hline 420 & -0.01371 & 0.001003 & 0.0004748 & 0.0 & +0.02220 \\
\hline 422 & -0.05564 & 0.003270 & -0.001434 & -0.001434 & +0.09237 \\
\hline 440 & 0.02389 & -0.01080 & -0.01080 & 0.0 & -0.4888 \\
\hline 442 & 0.02860 & -0.01005 & -0.01005 & -0.001026 & -0.5128 \\
\hline 444 & 0.03572 & -0.01888 & -0.01888 & -0.01888 & -1.569 \\
\hline 511 & -0.006659 & 0.05452 & 0.0008155 & 0.0008155 & +0.1505 \\
\hline 531 & -0.005499 & -0.0003738 & -0.0004552 & -0.0003732 & -0.02418 \\
\hline 533 & -0.02660 & -0.0006812 & -0.003463 & -0.003463 & -0.2126 \\
\hline 551 & 0.01551 & -0.007550 & -0.007550 & -0.0004523 & -0.5450 \\
\hline 555 & 0.02162 & -0.01188 & -0.01188 & -0.01188 & -1.5435 \\
\hline 600 & -0.003343 & 0.00455 & 0.0 & 0.0 & +0.1638 \\
\hline 620 & -0.002266 & 0.002211 & 0.0009362 & 0.0 & $\begin{array}{l}-0.09603 \\
+\end{array}$ \\
\hline 622 & -0.005262 & 0.002904 & -0.0001848 & -0.0001848 & $\begin{array}{l}+0.1283 \\
\end{array}$ \\
\hline 640 & -0.004214 & -0.0009978 & -0.0008832 & 0.0 & -0.06929 \\
\hline
\end{tabular}

a Using Ref. 6 force constants.

within the range of the potential bonds of atoms in the fifth shell, were taken into account but atoms beyond the fifth shell were not allowed to move from their perfect lattice positions. The displacements were obtained using an iterative procedure.
The results of the $\mathrm{Na}(1)$ displacements obtained in this fashion are compared with the analogous results of lattice statics in Table $\mathbf{X}$.

It is apparent from Table $\mathrm{X}$ that the two methods give comparable results but the discrepancies that exist,

TABLE VII. Displacement and interaction energy results for $\mathrm{Rb} . \mathrm{a}$

\begin{tabular}{|c|c|c|c|c|c|}
\hline $\begin{array}{l}\text { Neighbor } \\
\left(L_{1}, L_{2}, L_{3}\right)\end{array}$ & $\begin{array}{c}\text { Interaction } \\
\text { energy in } \mathrm{eV} \\
\text { between }(0,0,0) \\
\text { and }\left(L_{1}, L_{2}, L_{3}\right) \\
\text { vacancies }\end{array}$ & \multicolumn{3}{|c|}{$\begin{array}{l}\text { Displacement components of neighbors } \\
\text { around a vacancy (units of } 2 a \text { ) }\end{array}$} & $\begin{array}{c}|\zeta|\left\{L_{1}{ }^{2}+L_{2}{ }^{2}+L_{3}{ }^{2}\right\} \\
\text { + indicates } \\
\text { outward } \\
\text { relaxation } \\
\text { - indicates } \\
\text { inward } \\
\text { relaxation }\end{array}$ \\
\hline 111 & 0.05047 & -0.1117 & -0.1117 & -0.1117 & -0.5801 \\
\hline 200 & -0.2821 & 0.1259 & 0.0 & 0.0 & $\begin{array}{r}-0.5035 \\
+\end{array}$ \\
\hline 220 & 0.2243 & -0.03352 & -0.03352 & 0.0 & -0.3792 \\
\hline 222 & 0.09942 & -0.06509 & -0.06509 & -0.06509 & -1.353 \\
\hline 311 & -0.1697 & 0.01553 & 0.008576 & 0.008576 & +0.2167 \\
\hline 331 & 0.09037 & -0.01857 & -0.01857 & -0.001147 & -0.4995 \\
\hline 333 & 0.07097 & -0.03919 & -0.03919 & -0.03919 & -1.833 \\
\hline 400 & 0.06430 & 0.02140 & 0.0 & 0.0 & +0.3424 \\
\hline 420 & -0.01975 & 0.001033 & 0.0005808 & 0.0 & +0.02370 \\
\hline 422 & -0.07870 & 0.004450 & -0.0006400 & -0.0006400 & $\begin{array}{l}+0.1090 \\
\end{array}$ \\
\hline 440 & 0.02939 & -0.01317 & -0.01317 & 0.0 & -0.5958 \\
\hline 442 & 0.04008 & -0.01212 & -0.01212 & -0.001023 & -0.6182 \\
\hline 444 & 0.04688 & -0.02402 & -0.02402 & -0.02402 & -1.997 \\
\hline 511 & -0.007547 & 0.007120 & 0.001598 & 0.001598 & +0.2017 \\
\hline 531 & -0.006631 & -0.0004991 & -0.0003140 & -0.0004939 & -0.02692 \\
\hline 533 & -0.03904 & -0.0005821 & -0.003717 & -0.003717 & -0.2274 \\
\hline 551 & 0.01970 & -0.009241 & -0.009241 & -0.0005027 & -0.6670 \\
\hline 555 & 0.02898 & -0.01513 & -0.01513 & -0.01513 & -1.9656 \\
\hline 600 & 0.0002282 & 0.005874 & 0.0 & 0.0 & +0.2115 \\
\hline 620 & -0.003138 & 0.002702 & 0.001270 & 0.0 & +0.1194 \\
\hline 622 & -0.006180 & 0.003881 & 0.0002158 & 0.0002158 & +0.1713 \\
\hline 640 & -0.005879 & -0.001226 & -0.0008727 & 0.0 & -0.07826 \\
\hline
\end{tabular}

- Using Ref. 6 force constants. 
TABLE VIII. Displacement and interaction energy results for Cs. ${ }^{\mathrm{a}}$

\begin{tabular}{|c|c|c|c|c|c|}
\hline \multirow{2}{*}{$\begin{array}{l}\text { Neighbor } \\
\left(L_{1}, L_{2}, L_{3}\right)\end{array}$} & \multirow{2}{*}{$\begin{array}{c}\text { Interaction } \\
\text { energy in } \mathrm{eV} \\
\text { between }(0,0,0) \\
\text { and }\left(L_{1}, L_{2}, L_{3}\right) \\
\text { vacancies }\end{array}$} & \multicolumn{3}{|c|}{$\begin{array}{l}\text { Displacement components of neighbors } \\
\text { around a vacancy (units of } 2 a \text { ) }\end{array}$} & \multirow[t]{2}{*}{$\begin{array}{c}|\zeta|\left\{L_{1}{ }^{2}+L_{2}{ }^{2}+L_{3}{ }^{2}\right\} \\
\text { + indicates } \\
\text { outward } \\
\text { relaxation } \\
\text { - indicates } \\
\text { inward } \\
\text { relaxation }\end{array}$} \\
\hline & & $\zeta_{1}$ & $\zeta_{2}$ & $\zeta_{3}$ & \\
\hline $\begin{array}{l}111 \\
200 \\
220 \\
222 \\
311 \\
331 \\
333 \\
400 \\
420 \\
422 \\
440 \\
442 \\
444 \\
511 \\
531 \\
533 \\
551 \\
555 \\
600 \\
620 \\
622 \\
640\end{array}$ & $\begin{array}{c}0.07847 \\
-0.3015 \\
0.2505 \\
0.1083 \\
-0.1939 \\
0.1024 \\
0.07753 \\
0.08562 \\
-0.02319 \\
-0.08928 \\
0.03013 \\
0.04489 \\
0.05198 \\
-0.007998 \\
-0.006523 \\
-0.04470 \\
0.02048 \\
0.03222 \\
0.003869 \\
-0.004142 \\
-0.006496 \\
-0.006217\end{array}$ & $\begin{array}{c}-0.1202 \\
0.1409 \\
-0.03601 \\
-0.06998 \\
0.01652 \\
-0.01933 \\
-0.04203 \\
0.02435 \\
0.0006899 \\
0.004519 \\
-0.01357 \\
-0.01250 \\
-0.02562 \\
0.007751 \\
-0.0007836 \\
-0.0008001 \\
-0.009496 \\
-0.01603 \\
0.006269 \\
0.002802 \\
0.004173 \\
-0.001437\end{array}$ & $\begin{array}{l}-0.1202 \\
0.0 \\
-0.03601 \\
-0.06998 \\
0.01016 \\
-0.01933 \\
-0.04203 \\
0.0 \\
0.0001756 \\
-0.0001448 \\
-0.01357 \\
-0.01250 \\
-0.02562 \\
0.002086 \\
-0.0004776 \\
-0.003715 \\
-0.009496 \\
-0.01603 \\
0.0 \\
0.001303 \\
0.0005176 \\
-0.0009823\end{array}$ & $\begin{array}{l}-0.1202 \\
0.0 \\
0.0 \\
-0.06998 \\
0.01016 \\
-0.001511 \\
-0.04203 \\
0.0 \\
0.0 \\
-0.0001448 \\
0.0 \\
-0.001078 \\
-0.02562 \\
0.002086 \\
-0.0006252 \\
-0.003715 \\
-0.0005219 \\
-0.01603 \\
0.0 \\
0.0 \\
0.0005176 \\
0.0\end{array}$ & $\begin{array}{l}-0.6248 \\
+0.5637 \\
-0.4074 \\
-1.4545 \\
+0.2409 \\
-0.5202 \\
-1.965 \\
+0.3896 \\
+0.01424 \\
+0.1086 \\
-0.6140 \\
-0.6376 \\
-2.130 \\
+0.2239 \\
-0.03886 \\
-0.2285 \\
-0.6854 \\
-2.0827 \\
+0.2293 \\
+0.1236 \\
+0.1864 \\
-0.0905\end{array}$ \\
\hline
\end{tabular}

Asing Ref. 6 force constants.

particularly in the case of the first two neighbors, are somewhat disturbing. In view of the magnitudes of the displacements, it does not seem to us that (except possibly in the case of the first neighbors) the anharmonic corrections will be very significant; thus we are unable to account for this discrepancy. However, given that anharmonic effects are indeed unimportant, the method of lattice statics is in principal exact and its results are thus more reliable than those of a truncated iterative procedure.

No similar comparison can be made for the displacements calculated using the force constants given in Refs. 6 and 7 since no displacement calculations were performed by these authors. Moreover, since none of the metals considered is isotropic, it is not possible to compare the displacements predicted by lattice statics with those required by isotropic elasticity. However, it is to be expected that in any given crystallographic direction, ${ }^{4,5}$ the displacements of atoms far from the defect will fall off inversely as the square of their distance from the defect. The direction along which we have results for the greatest number of lattice sites is the $\langle 111\rangle$ direction and we indeed find that for each of the cases

TABLE IX. Dilatations and relaxation energies associated with a single vacancy in $\mathrm{Na}, \mathrm{K}, \mathrm{Rb}$, and $\mathrm{Cs}$. ( $\Delta V$ is given in atomic volumes, $E_{R}$. in

\begin{tabular}{ccccccc}
\hline & $\mathrm{Na}(1)$ & $\mathrm{Na}(2)$ & $\mathrm{K}(1)$ & $\mathrm{K}(2)$ & $\mathrm{Rb}$ & $\mathrm{Cs}$ \\
\hline$\Delta V$ & -0.696 & -1.82 & -0.322 & -1.61 & -1.933 & -2.12 \\
$E_{R}$ & -0.045 & -0.177 & -0.031 & -0.339 & -0.409 & -0.435 \\
\hline \hline
\end{tabular}

treated, the values of $\zeta R^{2}$ for $(3,3,3),(4,4,4)$, and $(5,5,5)$ are resonably close to each other, although not close enough to indicate that the true limiting value has been reached.12a It is of interest that, regardless of the potential used, the values of $\zeta R^{2}$ are much larger along $\langle 111\rangle$ directions than along any other class of directions.

The present calculated displacements about vacancies in the bcc alkali metals are considerably larger than those calculated by the same method for vacancies in the fcc metals, $\mathrm{Cu}$ and $\mathrm{Al}$. The much larger displacements found in the cases of $\mathrm{Na}(2)$ and $\mathrm{K}(2)$ relative to those obtained for $\mathrm{Na}(1)$ and $\mathrm{K}(1)$ can be attributed to the steeper potentials of Ref. 6 at first-, second-, and third-neighbor sites.

Using the Method of Long Waves, we have calculated the theoretical values of $C_{11}$ and $C_{44}$ for $\mathrm{Na}(1)$ and $\mathrm{Na}(2)$ and they are essentially the same for both

TABLE X. Comparison of $\mathrm{Na}(1)$ displacements (in $\%$ of normal separation distance).

\begin{tabular}{ccc}
\hline Neighbor & Reference 1 & Present work \\
\hline 111 & $-4.77^{\mathrm{a}}$ & -5.86 \\
200 & $+2.66^{\mathrm{b}}$ & +3.18 \\
220 & -0.62 & -0.95 \\
311 & +0.44 & +0.32 \\
222 & -0.86 & -1.43 \\
\hline
\end{tabular}

- sign indicates inward displacement.

12. Footnote added in proof. Subsequent work has shown that this is not the true asymptotic limit, which is only reached at a much greater distance from the defect. 
models. Thus we can infer that the strength of the vacancy is sensitive to the assumed interatomic potential in a manner very different from the way in which the dynamical matrix depends on this potential. In particular, it appears that the strength of the vacancy is very strongly dependent on the form of the interatomic potential at the first- and second-neighbor positions.

$\mathrm{It}$ is also apparent that in the cases of $\mathrm{Rb}$ and $\mathrm{Cs}$, the displacement of close neighbors of the vacancy are very large, as are the corresponding dilatations and relaxation energies. Thus it is likely that anharmonic effects will be significant for the closest neighbors to the defect. At a later stage we hope to investigate the possibility of including such effects. This does not seem to us to be profitable until the interatomic potential and its higher derivatives are more reliably defined.

As regards the calculated strain-field interaction energies, the most interesting result is certainly that the most stable configuration for the di-vacancy turns out to be the second-nearest-neighbor defect configuration. This conclusion is valid for all models and for all four metals, although the actual magnitudes of the interaction energies vary widely.

Finally, it should be noted that the value of the relaxation energy calculated for $\mathrm{Na}(1)$ in the present work is only about two-thirds of the value of $-0.071 \mathrm{eV}$ reported in Ref. 1. As a check on the validity of Eq. (19) we have calculated the relaxation energy of $\mathrm{Na}$ using the Morse potential and displacements due to Girifalco and Weizer ${ }^{13}$ and have obtained a value quite close to that calculated by Wynblatt and Gjostein ${ }^{14}$ using the same potential. However, if we use the displacements given by Shyu et al. ${ }^{1}$ instead of our displacements in Eq. (19) we obtain a value of the relaxation energy which is almost exactly half that quoted by these

${ }^{13}$ L. A. Girifalco and V. G. Weizer, J. Phys. Chem. Solids 12, 260 (1960).

${ }_{14}$ P. Wynblatt and W. A. Gjostein, J. Phys. Chem. Solids 28, 2108 (1967). authors. This discrepancy could be accounted for if these authors neglected to perform the division by two indicated in Eq. (9) of their paper.

\section{SUMMARY}

We have used the method of lattice statics to calculate the lattice distortion produced by single vacancies in $\mathrm{Na}, \mathrm{K}, \mathrm{Rb}$, and $\mathrm{Cs}$, and have used the same technique to find the strain-field interaction energies between various divacancies in these metals. We have used the displacements so obtained to find the dilatations and relaxation energies associated with single vacancies in each case. These calculations have been done using three distinct model interatomic potentials. ${ }^{1,6,7}$

We find a large variation in the distortions and relaxation energies associated with the potentials of Refs. 1 and 7 as compared with those resulting from the model potentials of Ref. 6 . This would appear to imply that at least one of the two alternative potentials in each of the cases of $\mathrm{Na}$ and $\mathrm{K}$ is unphysical.

The validity of the results in the present paper is limited by the accuracy of the potentials presently available. This fact does not detract in any way from the validity, accuracy and usefulness of the method of lattice statics upon which we have based all of our computations and which provides a logical and practical approach to the calculation of distortions produced by single defects under any assumed potential. When the true interatomic potentials in the alkali metals are reliably determined, a repetition of the present calculations can be performed and will yield correspondingly accurate displacements, interaction energies, relaxation energies, and dilatations.

Essentially the use of lattice statics removes any computational uncertainty from these quantities, and the residual uncertainty, at present large, is entirely due to our present imperfect knowledge of the proper interatomic potentials to use. 